\title{
Steroid-induced rosacea- a study from a tertiary care Hospital of Eastern Nepal
}

\author{
Chaudhary MK' ${ }^{1}$ Agrawal $\mathbf{S}^{2}$ \\ ${ }^{1}$ Consultant Dermatologist, Swara Skin and Hair Clinic, Itahari, Nepal; ${ }^{2}$ Professor, Department of Dermatology, BP \\ Koirala Institute of Health Sciences, Dharan, Nepal
}

\begin{abstract}
Introduction: Topical corticosteroids (TCS) are useful for treatment of various dermatological conditions in all age groups. Due to its cosmetic application TCs misuse is intertwined with fairness creams in our colour conscious society where people are obsessed with fair colour.

Objectives: To find the clinical presentations of steroid induced rosacea like-dermatitis on the face and to evaluate the purpose behind misusing TCs on the face.

Materials and methods: Descriptive prospective questionnaire-type case series study was approved by Institutional Ethical Committee of BPKIHS which involved 205 patients diagnosed as Steroid Induced Rosacea who consulted Dermatology OPD between March to June 2017.

Results: Among 205 patients enrolled, majority were from second to fourth decade (87.31\%) of their life and maximum being females i.e. $91.2 \%$. Around $97 \%$ of the study population were unaware of side effects of TCs. Most patients use Mometasone fuorate (58.04\%) for melasma (88.29\%). Friends acted as source of prescription (29.75\%), followed by Pharmacist (24.88\%) \& Beauty centers (20\%). Dermatologists prescribed TCs in only $6.34 \%$ cases. Sun exposure was identified as the triggering factor for steroid induced rosacea in $73.17 \%$ patients. Cheek was the commonest site involve in $92.19 \%$, followed by forehead $(65.36 \%)$, perioral area $(46.83 \%)$, and chin $(9.75 \%)$. Mixed type of lesions was the commonest lesion found in $52.20 \%$ patients, followed by diffuse facial erythema (36.09\%), \& telangiectasia (33.17\%).

Conclusion: TCs misuse in the younger people for the sake of fairer skin with little or no knowledge about the adverse effects of this medication should be stopped.
\end{abstract}

Key words: Adrenal Cortex Hormones; Beauty; Mometasone Furoate; Telangiectasis

\section{Introduction}

T opical corticosteroids (TCS) are useful for treatment of various dermatological conditions in all age groups. ${ }^{1}$ The effects and adverse effects of topical corticosteroids depends on the chemical structure, vehicle, factors related to site of application, frequency and method of application. Due to the hyperproliferative effects of TCs atrophy, striae, telengiectasia, purpura, bruising and ulceration may occur. $^{2}$

Funding: No

Conflict of Interest: No

\section{Address of Correspondence}

Manoj Kumar Chaudhary

Ex Assistant Professor, Department of Dermatology, BP Koirala Institute of Health Sciences, Dharan, Nepal

Swara Skin and Hair Clinic, Itahari, Phone No: 9852820760

E-mail: manojchaudhary12@live.com
The chronic misuse of TCS on the face may produce a clinical condition which was described by various names, like light sensitive seborrhoeid, ${ }^{3}$ perioral dermatitis, ${ }^{4}$ rosacea-like dermatitis, ${ }^{5}$ steroid rosacea, ${ }^{6}$ steroid dermatitis resembling rosacea, ${ }^{7}$ and steroid induced rosacea-like dermatitis. ${ }^{8}$

\section{Submitted: $2^{\text {nd }}$ December 2020 \\ Accepted: $25^{\text {th }}$ January 2021 \\ Published: $20^{\text {th }}$ February 2021}

How to cite this article

Chaudhary MK, Agrawal S. Steroid-induced rosacea- a study from a tertiary care Hospital of Eastern Nepal. Nepal Journal of Dermatology, Venereology \& Leprology 2021;19(1):25-9. https://doi.org/10.3126/njdvl.v19i1.35952.

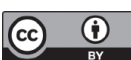

Licensed under CC BY 4.0 International License which permits use, distribution and reproduction in any medium, provided the original work is properly cited. 
A growing menace is the injudicious prescribing of TCs by non-dermatologists (particularly general practitioners, practitioners of alternative medicine and quacks) and laymen form a bulk population to "advise" patients regarding TCs. This cosmetic misuse of TCs is global and has been the subject of studies mainly from Africa, ${ }^{9}$ Asia ${ }^{10-12}$ and even developed countries like the USA are facing this problem. ${ }^{13}$ However, data on clinical use and misuse of topical corticosteroids in Nepal are sparse.

The aim of the present study was to document the clinical presentations of steroid induced rosacealike dermatitis and to evaluate the purpose behind misusing TCs on the face. This would help in planning the protocol for awareness program for public and doctors of all specialties and major steps for irrational topical steroid combinations.

\section{Materials and methods}

This descriptive prospective questionnaire-type case series study involved all patients clinically diagnosed as Steroid induced rosacea in the Department of Dermatology and Venereology, BPKIHS between March 2017 to June 2017 with prior informed and written consent. Ethical clearance was taken from the institutional ethical committee.

A questionnaire eliciting demographic variables, characteristics of TC use, potency, frequency, duration and reason of using the drug were administered to all eligible patients. Patients were also asked who had prescribed/recommended the drug [patient himself, friend, family members (i.e. over-the counter), pharmacist, paramedical personnel, physician/general practitioner, other specialist or dermatologist] and knowledge about the side-effects of the drugs before use.

A detailed cutaneous examination was performed to detect the findings related to abuse of topical corticosteroids. Medical photographic documentation of the patients were done after taking consent from each patient after full explanation of the goals and the nature of the study. Counseling and treatment of TC adverse effects were given to each patient.

Descriptive statistical analysis was done by using scientific calculator. The baseline characteristics of the study patients were expressed as numbers and percentages for categorical variables.
A special questionnaire was designated to include all clinical data like demographics, age of patient at onset of the disease, duration of the disease, symptoms and signs of the disease. A particular attention was given to corticosteroid therapy regarding the type, potency, duration of therapy, purpose, and the source of its use.

\section{Results}

A total of 205 clinically diagnosed cases of Steroid Induced Rosacea were enrolled in this study among them the major population were female (91.2\%) and remaining males $(8.8 \%)$. Majority of the study population were from second to fourth decade (87.31\%), followed by the age group of $41-50$ yrs (10.73\%), $11-20$ yrs (1.46\%) and least were of $\geq 50$ yrs $(0.46 \%)$. Among the study population $39.51 \%$ had received secondary level education followed by Primary level (25.85\%), University Level (20\%). Only $2.92 \%$ of the study population were aware of the side effects of TCs use. Majority of the population had used Mometasone fuorate (58.04\%), followed by Betamethasone valerate (14.63\%) and Clobetasol propionate (3.90\%). In this study the reason for using topical corticosteroids were Melasma 181 (88.29\%), followed by Acne 16 (7.80\%), Fairness 11 (5.36\%) and Freckles 3 (1.46\%). It was used for other purposes like pigmentation and nonspecific lesions by 4 (1.95\%) patients. Most of the study population had friends as source of prescription 61 (29.75\%), followed by Pharmacist i.e. 51 (24.88\%), Beauty centers i.e. 41 (20\%). Self prescription was seen in 17 (8.29\%) patients. Dermatologist and general physician prescribed topical corticosteroids in only $13(6.34 \%)$ and $4(1.90 \%)$ patients. Relatives were source of prescription in 9 (4.39\%) while radio, TV and newspaper were in 11 (5.36\%) patients. Sun exposure (73.17\%) was the commonest triggering factor while no triggering factor was found in $20.97 \%$ of the study population. Other factors identified as triggering factors were stress, exercise, threading etc. Cheek was the commonest site involved in 188 (92.19\%), followed by fore head in 134 (65.36\%), perioral area in 96 (46.83\%) and chin in 20 (9.75\%). Among the different types of lesions during presentation, Mixed type of lesions was the commonest lesion found in 107 (52.20\%) patients, followed by diffuse facial erythema in 71 (36.09\%), telangiectasia in 68 (33.17\%). Other types of lesion were papular, oedema and papulopustular lesions. 
Table 1: Demographic Status of the patients

\begin{tabular}{|l|c|c|}
\hline Age Group (years) & Number & Percentage \\
\hline $11-20$ & 3 & 1.46 \\
\hline $21-30$ & 90 & 43.90 \\
\hline $31-40$ & 89 & 43.41 \\
\hline $41-50$ & 22 & 10.73 \\
\hline$\geq 50$ & 1 & 0.48 \\
\hline Gender & 18 & 8.8 \\
\hline Male & 187 & 91.2 \\
\hline Female & 13 & \\
\hline Education Level & 30 & 14.63 \\
\hline Illiterate/Uneducated & 53 & 25.85 \\
\hline Primary Level & 81 & 39.51 \\
\hline Secondary Level & 41 & 20 \\
\hline University Level & 13 & 20.97 \\
\hline Awareness of side effects & 13 & 73.17 \\
\hline No & 13 & 6.34 \\
\hline Yes & 199 & 9.97 .02 \\
\hline Type of topical \\
corticosteroid formulation \\
used
\end{tabular}

Table 2: Site of Involvement

\begin{tabular}{|l|c|c|}
\hline & Number & Percentage \\
\hline Cheek & 188 & 92.19 \\
\hline Forehead & 134 & 65.36 \\
\hline Perioral & 96 & 46.83 \\
\hline Chin & 20 & 9.75 \\
\hline Others & 52 & 25.36 \\
\hline
\end{tabular}

Table 3: Types of Lesions

\begin{tabular}{|l|c|c|}
\hline & Number & Percentage \\
\hline Diffuse facial erythema & 74 & 36.09 \\
\hline Oedema & 2 & 0.97 \\
\hline Papular Lesions & 3 & 1.46 \\
\hline Papulo-pustular Lesions & 1 & 0.49 \\
\hline Telangectasia & 68 & 33.17 \\
\hline Mixed type of Lesions & 107 & 52.20 \\
\hline
\end{tabular}

\section{Discussion}

Topical Corticosteroid (TC) uses for different skin conditions are evident since last many decades. Also its side effects and adverse effects are being faced by the users due to different reasons like long term use, improper use or other factors as well. Since the uses of TCs are so aberrant these days, the adverse events due to its misuse are more common in today's population.

In our study, we found that majority of the study population were from second to fourth decade $(87.31 \%)$ of their life and maximum being females i.e. $91.2 \%$. The reason behind young women being mostly affected by this problem can partly be explained by the higher prevalence of pigmentary disorder like melasma and also women promptly respond to their cosmetic needs.

Around $40 \%$ of the study population in our study attended secondary level education, followed by Primary level 53 (25.85\%) and University level 41 (20\%). And majority of the study population 199 (97.08\%) were unaware of the side effects. This shows that most of the population had lower level of education which might had led to the misuse of TCs.

Most of the patients used Mometasone furoate 119 (58.04\%), followed by Betamethasone valerate 30 (14.63\%), Clobetasol propionate 8 (3.90\%). Topical steroids like fucinolone acetonide and fluticasone propionate were used by $48(23.41 \%)$ patients. These are the topical drugs that are easily available over the counter in the pharmacy which can be purchased without the doctor's prescription here in Nepal. 
The reasons for using topical corticosteroids by the study population were Melasma 181 (88.29\%), followed by Acne 16 (7.80\%), Fairness 11 (5.36\%) and Freckles $3(1.46 \%)$, while 4 (1.95\%) patients used for other purposes like pigmentation and nonspecific lesions. Hameed AF (2013) also figured out that TCs misuse if common for melasma, freckles and actinic lichen planus to have fairer look as a mainstay for the clients to use TCs on their face. ${ }^{14}$

Majority of study population had Friends as source of prescription 61 (29.75\%), followed by Pharmacist i.e. 51 (24.88\%), Beauty centers i.e. 41 (20\%). Dermatologist and general physician prescribed topical corticosteroids in only $13(6.34 \%)$ and 4 (1.90\%) patients respectively. Relatives were source of prescription in 9 (4.39\%) while radio, TV and newspaper were in 11 (5.36\%) patients. This finding is similar to the study done by Chohan et al $^{15}$ in which friends, relatives and peers acted as source of prescription in $64 \%$ of the patients. These sorts of practice reflect the lack of regulatory oversight, social factors and influence of advertising strategies used by manufacturers and aggressive marketing to dupe the people.

Majority of the population identified sun exposure as the triggering factor for steroid induced rosacea in $150(73.17 \%)$ patients. Other triggering factors were identified as stress, exercise and threading in $13(6.34 \%), 2(0.97 \%)$ and $1(0.47 \%)$ respectively. No triggering factors were found in $43(20.97 \%)$ patients. Hameed $\mathrm{AF}^{14}$ also found sun exposure to be the most common triggering factor as the commonest one.

Cheek was the commonest site involved in 188 (92.19\%), followed by fore head in 134 (65.36\%), perioral area in 96 (46.83\%) and chin in 20 (9.75\%). TCs are most often used as fairness cream and face is the commonest site of misuse as it is cosmetically appreciable. $^{16}$

\section{References}

1. Burkhart G, Morrell D, Goldsmith L. Dermatological pharmacology. In: Brunton LL, Chabner BA, Knollmann BC, editors. Goodman and Gilman's The Pharmacological Basis of Therapeutics. 12th ed. New York: McGraw Hill; 2011. p. 1806-8.

2. Warner MR, Carnisa C. Topical corticosteroids. In: Wolvrton SE, editor. Comprehensive Dermatologic Drug Therapy. Philadelphia: Saunders Elsevier; 2007. p. 595-624.

3. Hengge UR, Ruzicka T, Schwartz RA, Cork MJ. Adverse effects of topical glucocorticosteroids.
Mixed type of lesions was the commonest lesion found in 107 (52.20\%) patients, followed by diffuse facial erythema in 71 (36.09\%), telangiectasia in 68 (33.17\%). Other types of lesion were popular, oedema and papulo-pustular lesions. Similar results were seen in the study done by Chohan et al, ${ }^{15}$ in which facial erythema was the commonest side effect, Beltrani et $\mathrm{al}^{17}{ }^{17}$ in which telengectasia was also the common side effect, and Bhat $\mathrm{YJ}$ et $\mathrm{al}^{18}$ who observed rosacea as common adverse effect.

\section{Recommendations}

1. TCs should not be used as a fairness cream and should be only made available to the patients under the Dermatologist's prescription.

2. The regulatory legislation should be made to stop over the counter use of the TCs.

3. Manufacturer's aggressive marketing to dupe the people to use TCs should be stopped.

\section{Conclusion}

TCs have been in use in the younger people for the sake of fairer skin with little or no knowledge about the adverse effects of this medication. So the aim of this study was to document the clinical presentations of steroid induced rosacea-like dermatitis and to evaluate the purpose behind misusing TCs on the face. This would help in planning the protocol for awareness program for public and doctors of all specialties and major steps for irrational topical steroid combinations.

\section{Acknowledgement}

I would like to acknowledge my guide and tutor Prof Sudha Agrawal for her constant and tireless support in the completion of the research. Also I would like to acknowledge my patients for all their information.
J Am Acad Dermatol 2006;54:1-15. https://doi. org/10.1016/j.jaad.2005.01.010

4. Frumess GM, Lewis HM. Light sensitive seborrheid. Arch Dermatol 1957;75:245-8.

5. Mihan R, Ayres S Jr. Perioral dermatitis. Arch Dermatol 1964;89:803-5. https://doi. org/10.1001/archderm.1957.01550140089014

6. Chen AYY, Zirwas MJ. Steroid-induced rosacea like dermatitis: case report and review of the literature. Cutis 2009;83:198204. 
7. Leyden J, Thew M, Kligman AM. Steroid rosacea. Arch Dermatol 1974;110:619-22. https://doi. org/10.1001/archderm.1974.01630100075019

8. Ljubojeviæ $S$, Basta-Juzba`sviæ A, Lipozen `eiæ J. Steroid dermatitis resembling rosacea: aetiopathogenesis and treatment. J Eur Acad Dermatol Venereol 2002;16:1216. https://doi. org/10.1046/j.1468-3083.2002.00388_2.x

9. Mahe A, Ly F, Aymard G, Dangou JM. Skin diseases associated with the cosmetic use of bleaching products in women from Dakar, Senegal. $\mathrm{Br}$ J Dermatol 2003;148:493-500. https://doi. org/10.1046/j.1365-2133.2003.05161.x

10. Lu H, Xiao T, Lu B, Dong D, Yu D, Wei H et al. Facial corticosteroid addictive dermatitis in Guiyang city, China. Clin Exp Dermatol 2009;35:618-21. https:// doi.org/10.1111/j.1365-2230.2009.03761.x

11. Agarwal A, Singhvi IJ, Bele D, Sharma K. Evaluation of steroids in face creams of different marketed brands. Int J Pharm Technol 2011;3:2480-6.

12. Rathi SK, Kumrah L. Topical corticosteroid-induced rosacea-like dermatitis: A clinical study of 110 cases. Indian J Dermatol Venereol Leprol 2011;77:42-

6. https://doi.org/10.4103/0378-6323.74974

13. Solomon BA, Glass AT, Rabbin PE. Tinea incognito and over the counter potent topical steroids. Cutis 1996;58:295-6.

14. Hameed AF. Steroid Dermatitis Resembling Rosacea: A clinical evaluation of 75 patients. ISRN Dermatology 2013;5:491376. https://doi. org/10.1155/2013/491376

15. Chohan SN, Suhail M, Salman S, Bajwa UM, Saeed $M$, Kausar $S$ et al. Facial abuse of topical steroids and fairness creams: a clinical study of 200 patients. J Pak Assoc Dermatol 2014;24(3):204-11.

16. Nnoruka E, Okoye OJ. Topical steroid abuse: its uses a depigmenting agent. Natl Med Assoc 2006;98:934-9.

17. Beltrani VS, Barsanti FA, Bielory L. Effects of glucocorticosteroids on the skin and eye. Immunol Allergy Clin North Am 2005;25:557-80. https://doi. org/10.1016/j.iac.2005.05.006

18. Bhat YJ, Manzoor S, Qayoom S. Steroid-induced rosacea: a clinical study of 200 patients. Indian J Dermatol 2011;56:30-2. https://doi. org/10.4103/0019-5154.77547. 